\title{
Gingival Depigmentation with Scalpel and Diode Laser
}

\author{
Amit Bhardwaj, Harpreet Singh Grover, Sanjay Lal
}

\begin{abstract}
The complaint of black gums is common particularly in patients having a very high smile line and possess esthetic problems for them. The degree of gingival pigmentation depends on melanoblastic activity. Various treatment options are in practice for depigmentation which includes scalpel gingivectomy, free gingival graft surgery, use of chemicals, such as $90 \%$ phenol and $95 \%$ alcohol, bur abrasion, electrosurgery, cryosurgery and lasers. Among the various techniques lasers offer a promising therapeutic option since it is simple, painless and predictable. Laser has been recognized as the most effective and reliable technique having many advantages as compared to other conventional treatment modalities. It provides a bloodless and painless surgery. Here, we report gingival depigmentation of 21-year-old female patient using scalpel and laser technique.
\end{abstract}

Keywords: Gingival depigmentation, Laser, Scalpel.

How to cite this article: Bhardwaj A, Grover HS, Lal S. Gingival Depigmentation with Scalpel and Diode Laser. World J Dent 2012;3(4):359-362.

\section{Source of support $\mathrm{Nil}$}

Conflict of interest: None declared

\section{INTRODUCTION}

Gingival health and appearance are essential components of an attractive smile. Color of gingiva depends on number and size of blood vessels, epithelial thickness, quantity of keratinization and pigments within epithelium like melanin, carotene, reduced hemoglobin and oxyhemoglobin. ${ }^{1}$ $M$ elanin is the most common natural pigment contributing to color of gums. ${ }^{2}$ Although melanin pigmentation of the gingiva is completely benign and does not present a medical problem, complaints of black gums are common particularly in patients having a very high smile line (gummy smile). Oral melanin pigmentation is well-documented in the literature and is considered to be multifactorial, whether physiological/pathological and can be caused by a variety of local and/or systemic factors ${ }^{3}$ including genetic, tobacco use, prolonged administration of certain drugs especially antimalarial agents and tricyclic antidepressants endocrine disturbance, Albright's syndrome, malignant melanoma, Peutz-J eghers syndrome, trauma, hemochromatosis, chronic pulmonary disease and racial pigmentation. ${ }^{4}$ This pigmentation may be seen across all the races ${ }^{5}$ and at any age ${ }^{6}$ and it is without gender predilection. ${ }^{7}$ High levels of oral melanin pigmentation are normally observed in individuals of A frican, East A sian or Hispanic ethnicity. It is generally agreed that pigmented areas are present only when melanin granules synthesized by melanocytes are transferred to the keratinocytes. This close relationship between melanocytes and keratinocytes was labeled by Fitzpatrick and B reathnach (1963) as the epidermal-melanin unit. $^{8}$ Demand for cosmetic therapy of gingival melanin pigmentation is common. Gingival depigmentation has been carried out using various nonsurgical and surgical procedures. Different treatment modalities which have been reported include bur abrasion, scraping, partial thickness flap, cryotherapy, electrosurgery, gingivectomy, gingivectomy with free gingival autografting, chemical agents, such as $90 \%$ phenol and $95 \%$ alcohol and lasers. Recently, laser ablation has been recognized as a most effective, pleasant and reliable technique.

\section{CASE REPORT}

A 21-year-old female reported to the Department of Periodontics and O ral Implantology of SGT D ental College and R esearch Institute, Gurgaon, with a chief complaint of black colored gingiva. Since, she was very conscious about her esthetic appearance, she wants to get it corrected. Intraoral examination revealed deeply pigmented gingiva from 1st premolar to 1st premolar in both maxillary and mandibular arches (Fig. 1). The pigmentation was esthetically displeasing and hence, gingival depigmentation procedure was planned only in maxillary arch, since the mandibular region was not visible during talking and smiling, etc. For right maxillary quadrant, scal pel technique for depigmentation was planned and for left maxillary quadrant, use of diode laser was planned. A ccordingly, phase I therapy including thorough scaling and root planning was performed and a routine blood examination of the patient was done which revealed all values within the normal limits.

\section{Procedure}

For right maxillary side, local anesthesia was infiltrated. B ard-Parker handle with a no. 15 blade was used to remove the pigmented layer (Fig. 2). Pressure was applied with a sterile gauze moistened in saline to control hemorrhage during the procedure. High volume suction was used near the tissue. For left maxillary side, topical anesthetic spray was used. Sirona diode laser having wavel ength 810 to $980 \mathrm{~nm}$ at 1.5 to $2 \mathrm{~W}$ att power in a continuous wave mode with flexible fiber optic quartz delivery system was used 


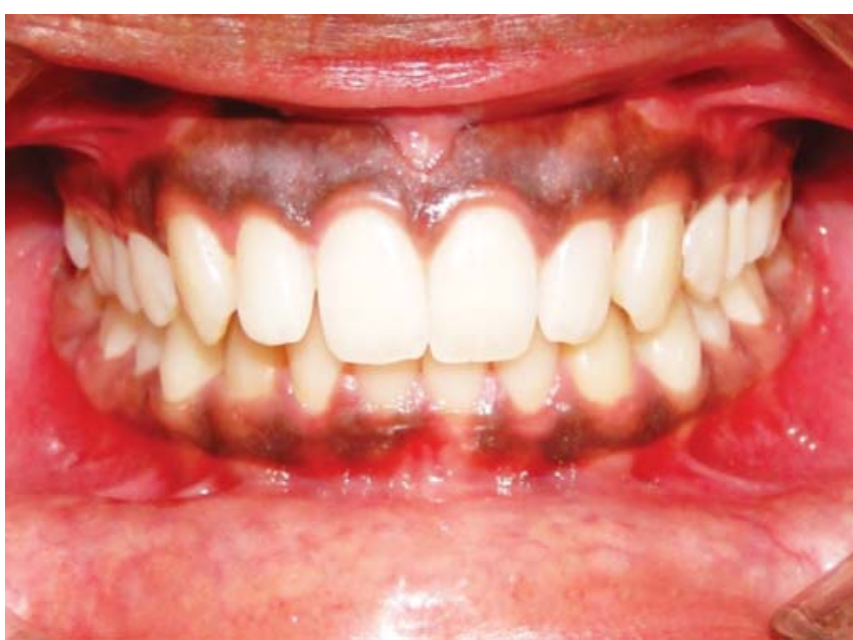

Fig. 1: Pigmentation on both maxillary and mandibular gingiva

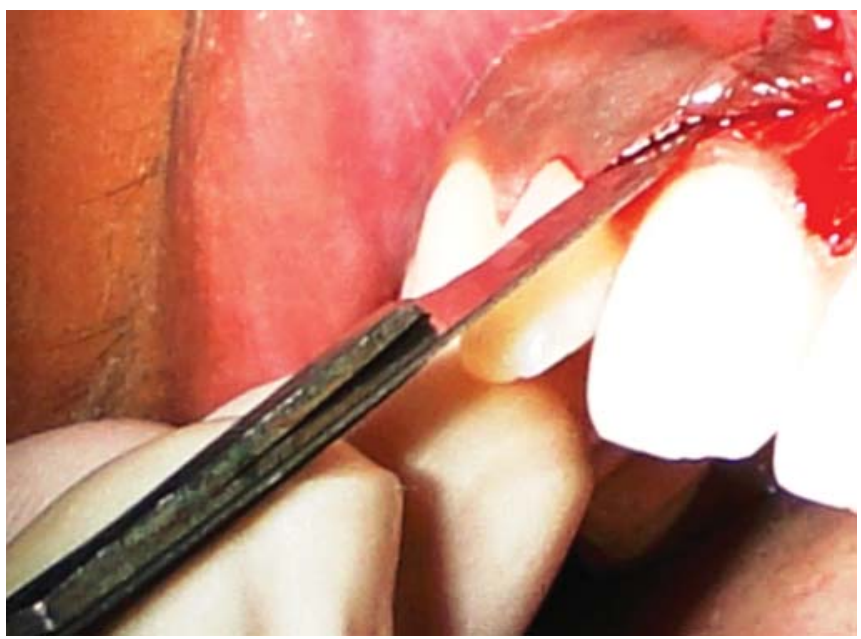

Fig. 2: Removal of pigmented layer using scalpel

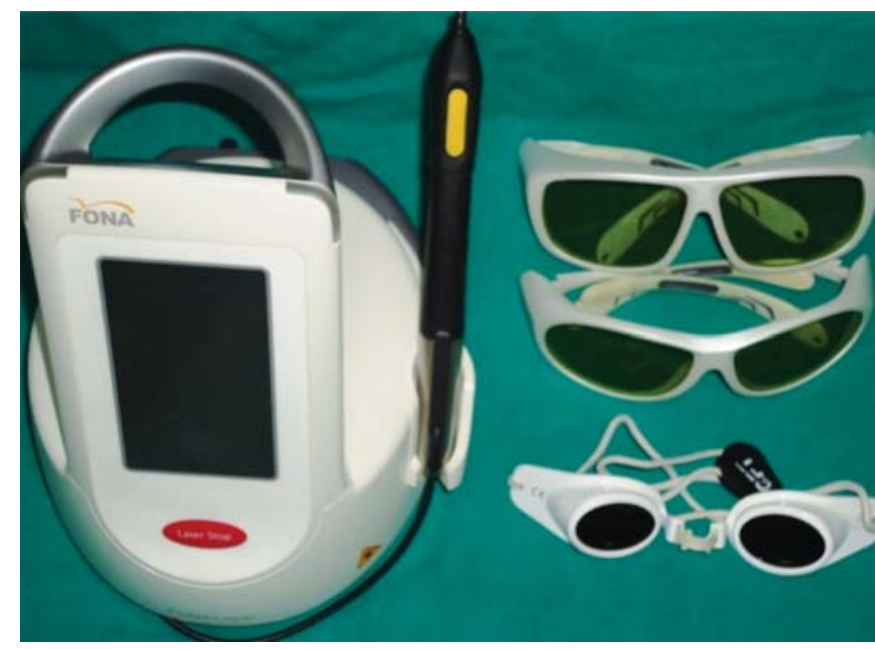

Fig. 3: Sirona diode laser unit

(Fig. 3). A fter the selected power settings were entered, the laser was activated. The procedure was performed in contact mode. The tip was held in light contact with the tissue and the procedure was performed with light sweeping brush

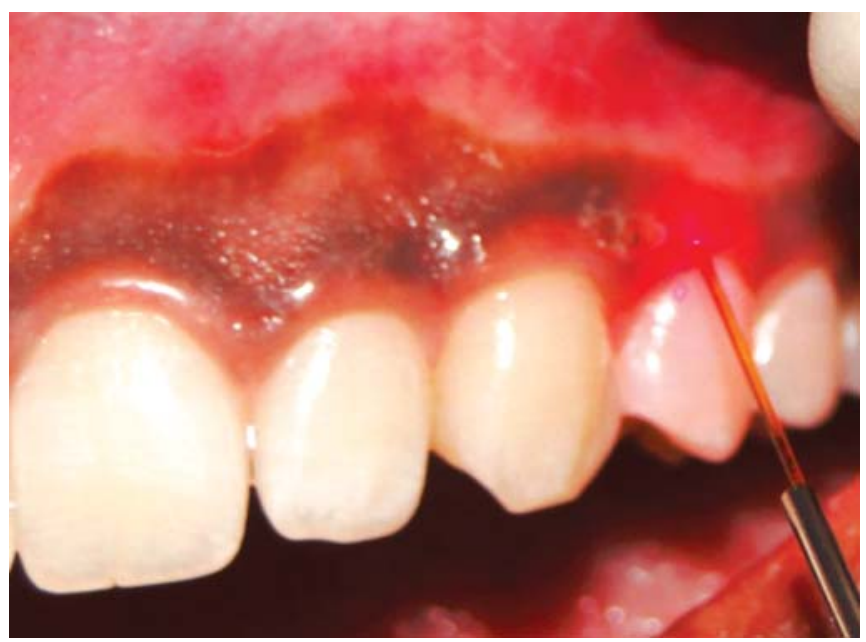

Fig. 4: Contact of laser tip with the pigmented tissue

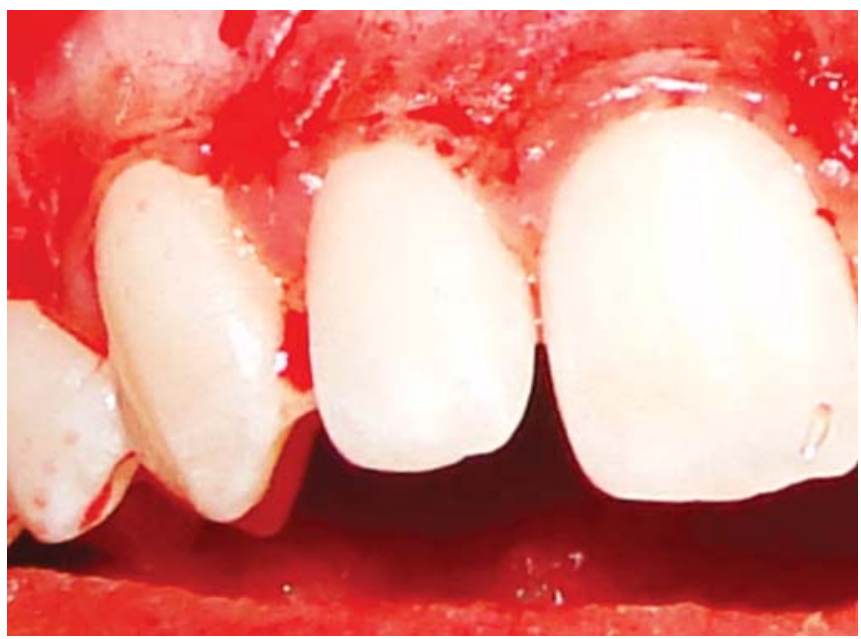

Fig. 5: Immediate postoperative site of scalpel technique

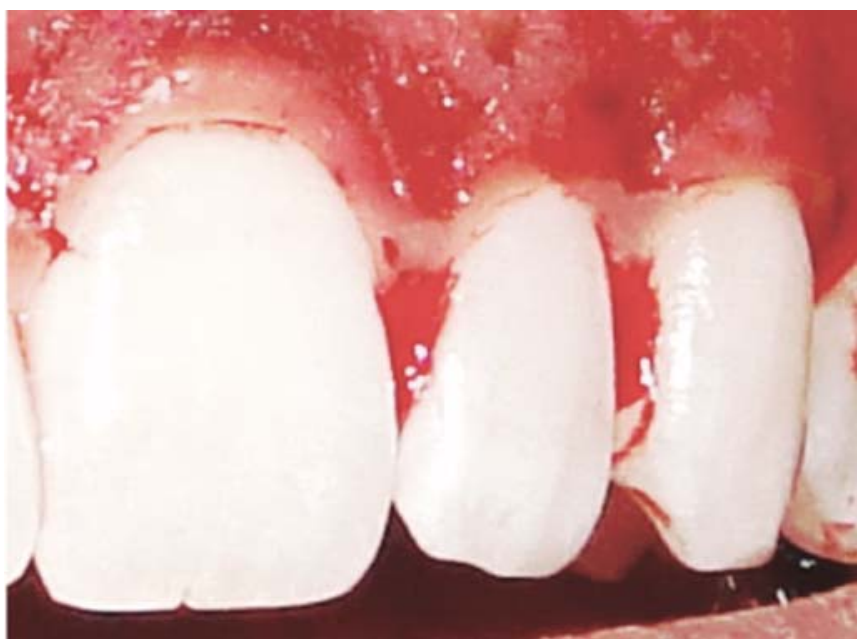

Fig. 6: Immediate postoperative site of laser technique

strokes (Fig. 4). High volume suction was used. The laser tip was directed to the target tissue until blister formation occurred. Blistered gingiva was scraped off with wet, saline moistened gauze to remove the epithelium containing 
G ingival Depigmentation with Scalpel and Diode Laser

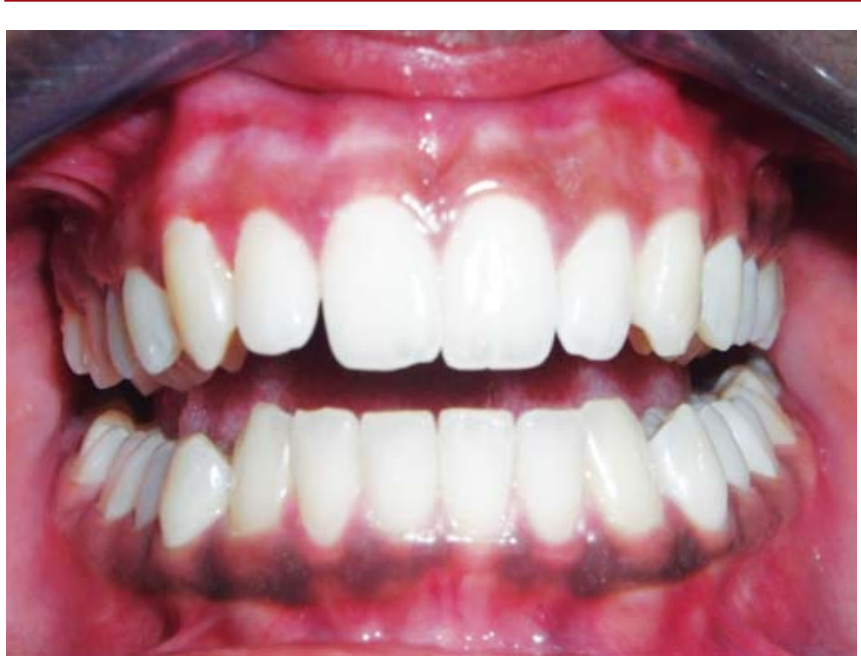

Fig. 7: Healing of both sides after 1 week

melanin pigmentation. No pain was experienced by the patient during the procedure. Patient was advised to take paracetamol $(650 \mathrm{mg})$ immediately after the procedure. Following the procedure no periodontal pack was given and no antibiotics were administered.

B oth sides were appeared normal immediately after the surgery (Figs 5 and 6 ). Patient was called on the 3rd day, when progressive healing of surgical site was seen. Patient did not report any pain or discomfort on the side treated with laser after the procedure. She felt little discomfort on the side treated with the scalpel but there was no pain on that side. A 1 week review appointment, complete healing of wound on both sides was seen (Fig. 7). At present, i.e. after 2 months, the pigmentation has not recurred. Patient is still on follow-up visits.

\section{DISCUSSION}

LASER is an acronym for Light Amplification by Stimulated Emission of Radiation, based on theories and principles first put forth by Einstein in the early 1900s. The first actual laser system was introduced by M aiman in $1960{ }^{9}$ Laser light is a man-made single-photon wavelength. The process of lasing occurs when an excited atom is stimulated to remit a photon before it occurs spontaneously. Stimulated emission of photons generates a very coherent, collimated, monochromatic ray of light. ${ }^{10}$ Clinical lasers are of two types: Soft and hard lasers. Soft lasers are claimed to aid healing and to reduce inflammation and pain. Its applications include frenectomies, ablation of lesions, incisional and excisional biopsies, gingivectomies, gingivoplasties, deepithelization, soft tissue tuberosity reductions, operculum removal, coagulation of graft donor sites and certain crown lengthening procedures. Surgical hard lasers, however, can cut both hard and soft tissues. Currently, numerous laser systems; both soft and hard lasers are available for dental use, like neodymium-doped yttrium-aluminum-garnet $(\mathrm{Nd} \text { :Y A G })^{11}$ carbon dioxide $\left(\mathrm{CO}_{2}\right),{ }^{12}$ semiconductor diode lasers, ${ }^{13}$ erbium-doped yttrium-al uminum-garnet ( $E r: Y A G$ ) laser.

There are many advantages of lasers over surgical procedures. A ccording to W igdor et al (1995), these include:

1. Dry and bloodless surgery,

2. Instant sterilization of the surgical site,

3. Reduced bacteremia,

4. Reduced mechanical trauma,

5. M inimal postoperative swelling and scarring and

6. M inimal postoperative pain.

All these above-mentioned advantages are evidently experienced in the above case. During procedure, there was no bleeding, which is al most always present when surgical approaches like bur abrasion, scraping, partial thickness flap or gingivectomy are used. A lso, postoperatively, no pain was experienced by the patient and no sw elling or any other signs of infection were noticed, whereas other alternative procedures have to be accompanied by administration of antibiotics and analgesics to minimize postoperative infection and pain. Especially, children and adolescents are among the best candidates for laser use because they are particularly bothered by pain, bleeding and extra postoperative office visits.

L asers can be used for patients to reduce anxiety or fear of drill. It provides a 'needle-free' approach or no anesthesia dentistry. A lso, laser dentistry requires less chairside time compared with more traditional treatments and hence results in more patient cooperation and more efficient dental practice. There is increased coagulation and a necrotic slough is formed over the surface of soft tissue after treating with lasers. There is no need of sutures and a faster and more comfortable healing is seen. Thus, it provides faster and better treatment of gum disease.

There are few disadvantages of laser. They are following:

1. Expensive,

2. Technique-sensitive,

3. N eed for eye protection for doctor, patient and assistant,

4. Hyperpigmentation can reappear in 6 months to 1 year time and

5. Tissue penetration from laser may cause thermal damage to underlying hard tissues.

\section{CONCLUSION}

For many intraoral soft tissue surgical procedures, the laser is a viable alternative to the scalpel. In the modern dental practice using laser technology, procedures can be accomplished with less invasive methods, a more relaxed 
appointment and less postoperative discomfort. L aser dental care is possible in all of the disciplines of dentistry. The public has an expectation that their dentist should be up-todate and wants the most modern, advanced care possible. The future of lasers in dentistry is promising, and new applications and procedures are being developed. Dentists and their staffs can successfully integrate the use of lasers into the everyday practice of dentistry.

\section{REFERENCES}

1. Mokeem SA. M anagement of gingival hyperpigmentation by surgical abrasion: R eport of three cases. Saudi D ent J 2006 SepDec;18(3):162-66.

2. Cicek $Y, E$ rtas $U$. The normal and pathological pigmentation of oral mucous membrane: A review. J Contemp Dent Pract 2003;4(3):76-86

3. Dummett CO. A classification of oral pigmentation. M il M ed 1962;127:839-40.

4. Leston JM, Santos A A, V arela-Centelles PI, Garcia JV, Romero M A , V illamor L P. O ral mucosa: V ariations from normalcy, Part II. Cutis 2002;69(3):215-17.

5. Dummet CO. Oral pigmentation. In: Dummet CO (Ed). First symposium on oral pigmentation (8th ed). J Periodontol 1960;31:356-60.

6. Page LR, Coro RE, Crawford BE, Giansanti JS, W eathers DR. The oral melanotic macule. Oral Sung Oral Med Oral Pathol 1977; 44:219-26

7. Trelles M A, V erkruysse W, Segui JM, U daeta A. Treatment of melanotic spots in the gingiva by argon laser. J O ral M axillofac Surg 1993;51:759-61.

8. Bergamaschi $0, K$ on S, Doine AI, Ruben MP. M elanin repigmentation after gingivectomy: A 5-year clinical and transmission electron microscopic study in humans. Int J Periodontics Restorative Dent 1993;13:85-92.

9. Maiman TH. Stimulated optical radiation in ruby. $\mathrm{N}$ ature 1960;187:493-94.

10. Clayman L, K uo P. L asers in maxillofacial surgery and dentistry. New Y ork: Thieme; 1997:1-9.

11. Atsawasuwan P, G reethong K, Nimmanon V. Treatment of gingival hyperpigmentation for esthetic purposes by $\mathrm{Nd}$ :Y AG laser: Report of 4 cases. J Periodontol 2000;71(2):315-21.

12. Nakamura $Y$, Hossain $M$, Hirayama $K, M$ atsumoto $K$. A clinical study on the removal of gingival melanin pigmentation with the $\mathrm{CO}_{2}$ laser. Lasers Surg M ed 1999; 25(2):140-47.

13. Y ousuf A, Hossain M, N akamura Y, Y amada Y, K inoshita J, $M$ atsumoto $K$. R emoval of gingival melanin pigmentation with the semiconductor diode laser: A case report. J Clin Laser M ed Surg 2000;18(5):263-66.

\section{ABOUT THE AUTHORS}

\section{Amit Bhardwaj (Corresponding Author)}

Senior Lecturer, Department of Periodontics, SGT Dental College Gurgaon, Haryana, India, e-mail: amitmds1980@ rediffmail.com

\section{Harpreet Singh Grover}

Professor and Head, Department of Periodontics, SGT D ental College, Gurgaon, Haryana, India

\section{Sanjay Lal}

Postgraduate Student, Department of Periodontics, SGT Dental College, Gurgaon, Haryana, India 